\title{
ON ABSTRACT PRUEFER TRANSFORMATIONS
}

\author{
DONALD C. BENSON AND KURT KREITH ${ }^{1}$
}

Abstract. The Pruefer transformation has been generalized to matrix differential equations by John Barrett, and Barrett's results have been partly extended to the case of functions which take values in a $B^{*}$-algebra by Einar Hille. By modifying Barrett's proof, generalizations in the $B^{*}$-algebra case become possible.

The Pruefer transformation for second order Sturn-Liouville equations was generalized to second order matrix differential equations by Barrett [1] and subsequently studied by Reid [2] and Etgen [3]. Hille [4] considered the case of differential equations involving functions which take their values in a $B^{*}$-algebra $B$ and obtained a partial extension of Barrett's results in this more general setting. The purpose of this note is to modify Barrett's original proof in such a way that it leads to generalizations of Hille's results.

As in [2], instead of considering a differential equation of SturmLiouville type, we shall consider the more general first order system

$$
Y^{\prime}=G(x) Z, \quad Z^{\prime}=-F(x) Y,
$$

where $G(x)$ and $F(x)$ are selfadjoint strongly continuous $B$-valued functions on some interval $[a, \infty)$ and derivatives are taken in the strong topology. We shall consider a solution $Y, Z$ of (1) satisfying

$$
Y(a)=0, \quad Z(a) \text { nonsingular. }
$$

Since (1) implies that $\left(Y^{*} Z-Z^{*} Y\right)^{\prime}=0$, we have $Y^{*} Z-Z^{*} Y=$ constant and, in view of $Y(a)=0$,

$$
Y^{*} Z-Z^{*} Y=0 .
$$

Thus our solution pair is conjoined in the terminology of Reid [2].

The Pruefer transformation for (1) consists of determining generalized sines and cosines $S(x)$ and $C(x)$ and a nonsingular $B$-valued function $R(x)$ such that the above solution $Y, Z$ of (1) admits the representation

Received by the editors January 20, 1970.

AMS 1969 subject classifications. Primary 3495; Secondary 3442.

Key words and phrases. Pruefer transformation, $B^{*}$-algebra, generalized sines and cosines, Picard existence theorem.

${ }^{1}$ Research supported by a grant of the National Science Foundation, NSF GP-11219. 


$$
Y(x)=S^{*}(x) R(x), \quad Z(x)=C^{*}(x) R(x) .
$$

The generalized sines and cosines are obtained as solutions $S(x ; a, Q)$ and $C(x ; a, Q)$ of

$$
S^{\prime}=Q C, \quad C^{\prime}=-Q S,
$$

satisfying $S(a)=0, C(a)=E$, where $E$ is the identity element of $B$ and $Q(x)$ is a selfadjoint strongly continuous function of $x$. It can be shown $[4$, Chapter 9.6] that for such solutions

$$
\begin{aligned}
& S S^{*}+C C^{*}=S^{*} S+C^{*} C=E, \\
& S C^{*}-C S^{*}=S^{*} C-C^{*} S=0 .
\end{aligned}
$$

The equations (1) and (4) yield

$$
\left(S^{*} R\right)^{\prime}=G C^{*} R, \quad\left(C^{*} R\right)^{\prime}=-F S^{*} R .
$$

Using (5), (6), and (7), these can be solved to yield

$$
\begin{aligned}
R^{\prime} & =\left(S G C^{*}-C F S^{*}\right) R, \\
Q R & =\left(C G C^{*}+S F S^{*}\right) R .
\end{aligned}
$$

Since $Z(a)=C^{*}(a) R(a)=R(a)$ and $Z(a)$ is nonsingular, (8) implies (see [4, p. 485]) that $R(x)$ is nonsingular for $a \leqq x<\infty$, and (9) becomes

$$
Q=C G C^{*}+S F S^{*} \text {. }
$$

This calculation shows that $(8)$ and $\left(9^{\prime}\right)$ are necessary conditions for the representation (4) to be valid, and a direct calculation [1] also shows that (8) and $\left(9^{\prime}\right)$ are sufficient. However, the difficulty is that since $S$ and $C$ both depend on $Q$, it is not obvious that $\left(9^{\prime}\right)$ has a solution. Indeed a major portion of Barrett's original proof consists of an existence theorem for $\left(9^{\prime}\right)$ which is established by iteration. This proof depends heavily on the fact that the $n \times n$ matrices $S(x)$ and $C(x)$ are bounded in the Frobenius-Wedderburn norm whiich assigns $\sqrt{ } n$ to the unit matrix. It is in this connection that a direct generalization to the $B^{*}$-algebra case fails (see $[4$, p. 483$]$ ).

The question of the solvability of (9) can be circumvented, however, by defining $S(x)$ and $C(x)$ to be solutions of

$$
S^{\prime}=\left(C G C^{*}+S F S^{*}\right) C, \quad C^{\prime}=-\left(C G C^{*}+S F S^{*}\right) S
$$

satisfying $S(a)=0, C(a)=E$. Our modification of Barrett's technique consists of the following observation.

Theorem 1. If $R(x), S(x)$ and $C(x)$ satisfy (8) and (5'), respectively, then the Pruefer transformation (4) is valid. 
Proof. We must verify that if $(8)$ and $\left(5^{\prime}\right)$ are satisfied, then

$$
\left(S^{*} R\right)^{\prime}=G C^{*} R \text { and }\left(C^{*} R\right)^{\prime}=-F S^{*} R .
$$

The first of these follows from the direct computation

$$
\begin{aligned}
S^{* \prime} R+S^{*} R^{\prime} & =C^{*}\left(C G C^{*}+S F S^{*}\right) R+S^{*}\left(S G S^{*}-C F C^{*}\right) R \\
& =\left(C^{*} C+S^{*} S\right) G C^{*} R=G C^{*} R,
\end{aligned}
$$

and the second follows from a similar computation.

The question remains as to whether (8) and $\left(5^{\prime}\right)$ have solutions. Since $\left(5^{\prime}\right)$ is just a first order differential equation in the Banach space $B \oplus B$ with norm

$$
\left\|\left(\begin{array}{l}
S \\
C
\end{array}\right)\right\|=|S|+|C|
$$

it follows from the generalized Picard existence theorem [5, Theorem 3.4.1] that $\left(5^{\prime}\right)$ has a local solution at $x=a$. Given $S(x)$ and $C(x)$ defined by $\left(5^{\prime}\right)$, (8) can clearly be solved for $R(x)$. This shows the existence of a local Pruefer transformation regardless of whether $S(x)$ and $C(x)$ are bounded in norm.

In order to obtain a Pruefer transformation valid on $[a, \infty)$, Hille makes the additional assumption that $Q(x)$ commutes with $\int_{a}^{x} Q(t) d t$ for all $a$ and $x$. With this hypothesis he shows that $|C| \leqq 1$ and $|S| \leqq 1$, and this fact leads to an existence proof for (5). The following theorem establishes other criteria for the boundedness of $|C|$ and $|S|$.

THEOREM 2. If $B$ is a $C^{*}$-algebra (that is an algebra of operators on a given Hilbert space $\mathfrak{S})$, then $|C| \leqq 1$ and $|S| \leqq 1$.

Proof. In the Hilbert space $\mathfrak{S}$, consider any solution $y, z$ of the system

$$
y^{\prime}=Q z, \quad z^{\prime}=-Q y
$$

satisfying $y(a)=0$ and $\|z(a)\|=1$. A direct calculation shows that

$$
[(y, y)+(z, z)]^{\prime}=0
$$

so that by the initial conditions

$$
(y, y)+(z, z) \equiv 1 \text {. }
$$

Now suppose that $Y, Z$ in $B(\mathfrak{S})$ satisfies $(5)$ and $Y(a)=0, Z(a)=E$. To show that $|Y| \leqq 1$ and $|Z| \leqq 1$ for all $x$, we note that for any constant vector $e$ of unit norm the vectors $y(x)=Y(x) e, z(x)=Z(x) e$ 
satisfy (10), so that by (11)

$$
\|Y(x) e\| \leqq 1, \quad\|Z(x) e\| \leqq 1
$$

for any $e \in \mathfrak{S}$ satisfying $\|e\|=1$. This completes the proof.

We remark that the above theorem applies to all complex $B^{*}$-algebras since they are known to be representable as $C^{*}$-algebras. It also applies to the real matrix case considered by Barrett [1].

Our final observation is that the boundedness of $|S|$ and $|C|$ leads to global solutions directly from $(8)$ and $\left(5^{\prime}\right)$ and that it is possible to use classical existence theory to circumvent the existence theorems devised by Barrett and Hille for the system consisting of (5) and $\left(9^{\prime}\right)$.

From the generalized Picard existence theorem (see [5, p. 67]) applied to the space $B \oplus B$ it follows that $\left(5^{\prime}\right)$ has a right maximal interval of existence $[a, b)$ where $a<b \leqq \infty$. On this interval $\left(5^{\prime}\right)$ has a solution $S, C$ but no solution exists on any interval $\left[a, b_{1}\right)$ with $b_{1}>b$.

If $\left(5^{\prime}\right)$ has the property that all solutions $S, C$ satisfy $|C| \leqq 1$, $|S| \leqq 1$, as is the case if $B$ is a $C^{*}$-algebra (Theorem 2 ), then one necessarily has $b=\infty$. This follows from the completeness of $B \oplus ß$ which makes it possible to give a direct generalization of the standard extension theorem for finite systems. (See [6, p. 15, Theorem 4.1].)

The above considerations are summarized in the following theorem and corollary.

THEOREM 3. If for any $c>$ a every solution of $\left(5^{\prime}\right)(S(a)=0, C(a)=E)$ on $[a, c)$ satisfies $|S(x)| \leqq 1$ and $|C(x)| \leqq 1$, then there exists a solution of $\left(5^{\prime}\right)(S(a)=0, C(a)=E)$ on the interval $[a, \infty)$.

COROLlary. If $B$ is a $C^{*}$-algebra then there exists a solution of $\left(5^{\prime}\right)$ $(S(a)=0, C(a)=E)$ on $[a, \infty)$.

\section{BIBLIOGRAPHY}

1. J. H. Barrett, A Prüfer transformation for matrix differential equations, Proc. Amer. Math. Soc. 8 (1957), 510-518. MR 19, 415.

2. W. T. Reid, A Prüfer transformation for differential systems, Pacific J. Math. 8 (1958), 575-584. MR 20 \#5913.

3. G. J. Etgen, Oscillatory properties of certain nonlinear matrix differential systems of second order, Trans. Amer. Math. Soc. 122 (1966), 289-310. MR 32 \#7834.

4. E. Hille, Lectures on ordinary differential equations, Addison-Wesley, Reading, Mass., 1969.

5. E. Hille and R. S. Phillips, Functional analysis and semi-groups, rev. ed., Amer. Math. Soc. Colloq. Publ., vol. 31 Amer. Math. Soc., Providence, R.I., 1957. MR 19, 664.

6. E. A. Coddington and N. Levinson, Theory of ordinary differential equatinns, McGraw-Hill, New York, 1955. MR 16, 1022.

University of California, Davis, California 95616 